\title{
Reynolds-number-dependent efficiency characterization of a micro-scale centrifugal compressor using non-conventional working fluids
}

\author{
Manuel Valdés, Andrés Sebastián*, Rubén Abbas \\ Universidad Politécnica de Madrid,c/ José Gutiérrez Abascal, 2, 28006 Madrid, Spain
}

\begin{abstract}
A B S T R A C T
The selection of working fluids other than air is a key issue in improving the efficiency of new thermodynamic cycles intended for low-to-moderate temperature small power plants The aim of this paper is to study whether the low efficiency typical of small turbomachinery is still a problem when using alternative fluids Based on a new design of power cycles named balanced hybrid Rankine-Brayton cycles, five different fluids were selected as potential working fluids: carbon dioxide, propane, isobutane, pentafluoroethane and sulfur hexafluoride

Dimensional analysis was used to compare the performances of a micro-scale centrifugal compressor working in homologous points where the efficiency variation depends only on the Reynolds-number (Re) The influence of Re on efficiency was calculated by means of four different methods for comparative purposes Numerical simulations were also carried out in order to validate the methodological approach proposed The results show the efficiency variations as a function of Re for increasing fluid densities All the nonconventional fluids studied provide better performance in terms of efficiency than air Particularly, isobutane and propane have been identified as potential working fluids candidates for the aforementioned innovative power cycle
\end{abstract}

\section{Introduction and background}

Power plants down-scaling has become a major challenge to promote a wider use of thermal renewable energy employing low-tomoderate temperature sources [1]. This may rise the contribution of solar [2], geothermal [3], biomass and waste energy [4] sources in future scenarios with increasing electricity distributed generation [5] and micro-combined heat and power systems [6]. However, a low temperature of the thermal source prevents the primary heat from being efficiently converted to electrical power. In such a case an extra effort should be paid to improve the thermodynamic cycles, which is the driving force of the authors' research in the renewable small-scale power plants field $[7,8]$. The selection of the working fluid is considered a key aspect in improving the efficiency of innovative cycles $[9,10]$. An important question when studying these cycles is whether the use of fluids other than air or steam may affect the efficiency of the polytropic processes, especially in low power cases using small-scale turbomachinery. This is precisely why the present paper is devoted to analyze these effects on micro-turbomachinery performance when using a novel power cycle based on working fluids other than air.

Micro-scale centrifugal compressors (MSCCs) are miniature turbocompressors (impeller diameter less than $30 \mathrm{~mm}$ ) working at ultra-high speeds (more than 200,000 $\mathrm{min}^{-1}$ ) [11]. This noteworthy down-scaling implies that the Reynolds-number (Re) could fall below a critical threshold under which viscous friction on the boundary layer becomes dominant according to Dixon [12]. This results in a certain efficiency loss, which severely affects the overall power cycle efficiency in smallscale power plants [13]. Conversely, at high Re, the boundary layers on the blades are generally turbulent and very thin, so they have little impact on the global flow field and its efficiency.

Organic Rankine cycles (ORCs) are considered a promising technology to drive forward micro and small-scale power cycles based on renewable heat sources $[14,15]$. Besides, novel conceptions of Brayton cycles using air [16] or fluids in supercritical conditions such as carbon dioxide $\left(\mathrm{CO}_{2}\right)[17,18]$ are also interesting for low power applications due to their high compactness and modularity [19]. In addition to power cycle applications, micro-scale centrifugal compressors may also be used in cryogenic reverse Brayton air refrigerators [20] and domestic heat pumps using 1,1,1,2-tetrafluoroethane (R134a), looking for better efficiencies than those given by currently used volumetric compressors $[21,22]$. Moreover, MSCCs are also of great interest for the air feeding of low-powered polymer electrolyte membrane fuel cells [23].

Rovira et al. proposed a novel balanced hybrid Rankine-Brayton (BHRB) power cycle $[24,25]$ oriented to low-to-moderate solar power plants. As its name suggests, features of both Rankine and Brayton cycles are coupled, i.e. the cycle includes a pump for the condensates

\footnotetext{
*Corresponding author.

E-mail address: andres.sebastian@upm.es (A Sebastián).
} 


\author{
Nomenclature \\ a speed of sound (m/s) \\ $b_{2} \quad$ impeller tip width (m) \\ $B_{\text {ref }} \quad$ Dietmann and Casey empirical coefficient ( ) \\ c absolute velocity (m/s), also chord (m) \\ $c_{f} \quad$ friction coefficient ( ) \\ $c_{p} \quad$ specific heat capacity $(\mathrm{kJ} / \mathrm{kgK})$ \\ D characteristic diameter (m) \\ $h \quad$ specific enthalpy $(\mathrm{kJ} / \mathrm{kg})$ \\ $L \quad$ characteristic length (m) \\ $\dot{m}_{s} \quad$ mass flow $(\mathrm{kg} / \mathrm{s})$ \\ $M_{c_{1}} \quad$ inlet absolute Mach number (\#) \\ $N \quad$ rotational sped $\left(\mathrm{min}^{-1}\right)$ \\ $p \quad$ pressure (bar) \\ $R \quad$ gas constant $(\mathrm{J} / \mathrm{kg} \mathrm{K})$ \\ Re Reynolds number ( ) \\ $T$ temperature (K) \\ $u \quad$ blade velocity $(\mathrm{m} / \mathrm{s})$ \\ $w \quad$ relative velocity $(\mathrm{m} / \mathrm{s})$
}

Acronyms

ATM Automatic Topology Meshing

B HRB Balanced Hybrid Rankine Brayton

CFD Computational Fluid Dynamics

\section{GCI Grid Convergence Index \\ MSCC Micro Scale Centrifugal Compressor \\ ORC Organic Rankine Cycle \\ SST Shear Stress Transport}

\section{Greek letters}

$\gamma \quad$ specific heat ratio ( )

$\eta \quad$ efficiency ( )

$\lambda \quad$ Moody diagram friction coefficient (Pa)

$\mu \quad$ dynamic viscosity (Pa $s$ )

$\nu \quad$ kinematic viscosity $\left(\mathrm{m}^{2} \mathrm{~s}\right)$

$\rho \quad$ fluid density $\left(\mathrm{kg} / \mathrm{m}^{3}\right)$

$\Phi \quad$ flow coefficient ( )

\section{Subscripts}

$0 x \quad$ total/stagnation conditions

1 impeller inlet

2 impeller outlet

3 diffuser outlet

crit critical

dif diffuser

imp impeller

sat saturation and a split compression stage. These high efficiency power cycles use non conventional fluids such as carbon dioxide $\left(\mathrm{CO}_{2}\right)$, isobutane, pro pane, pentafluoroethane (R125) and sulfur hexafluoride $\left(\mathrm{SF}_{6}\right)$. Hence, in order to characterize the compression stage for these B HRB cycles, this study is devoted to analyze the performance of a micro scale cen trifugal compressor using these alternative working fluids.

The novelty of this paper lies in the calculation of the efficiency variation that takes place when a small turbomachine such as a MSCC works with the potential fluids selected for the new design of B HRB power cycles. Similar works devoted to analyze the MSCC behavior in the particular compression stage proposed in these innovative power cycles have not been found in the scientific literature to the authors' knowledge. Therefore, this study can be of great interest to estimate the internal efficiency variation of turbomachinery when a certain fluid is selected for the maximization of the overall power cycle efficiency of $B$ HRB cycles. The idea behind this is to find out to what extent the low efficiencies of small turbomachinery usually working with air could be generalized to other fluids with different Reynolds numbers. The methodology developed in this paper is applied to a reference micro scale centrifugal compressor which is expected to work under B HRB conditions for the compression stage. Selected fluids for this analysis are the ones chosen for the B HRB power cycles and, also, air for comparative purposes. The obtained results will be used in the ex perimentation phase to be accomplished in the near future.

The methodology is described in Section 2. Dimensional analysis is used to compare the performances of a compressor working with two different fluids on homologous points with dynamical similarity con ditions. It is shown that if the head coefficient as well as both fluid and blade Mach numbers remain constant, the efficiency variation of the homologous points depends only on the Reynolds number variation. Besides, four efficiency correction methods are outlined including their own Reynolds number definition. The methodology ends with the de scription of the numerical model built to validate the approach pro posed. Performance calculations have been conducted in Section 3 to obtain the efficiency variations for air and the non conventional fluids selected as a function of Reynolds number. The Reynolds number was modified using different inlet pressures and thus different fluid entry densities. In addition, the rest of performance parameters variations are discussed. The paper ends with a synthesis of major conclusions in Section 4.

\section{Methodology}

The aim of this paper is to study whether the typical low efficiency of small turbomachinery is still a problem when using non conventional fluids. Hence, Reynolds number dependence on micro scale centrifugal compressors performance is investigated. A consistent methodology has been conceived in order to asses quantitatively this dependence. A set of homologous working points is obtained where efficiency changes depends only on Reynolds number (Section 2.1). Then efficiency cor rection methods are presented with their own Reynolds number defi nition (Section 2.2). Finally, a numerical model is presented to validate the methodological approach used (Section 2.3).

\subsection{Calculation of homologous points for different fluids through dimensional analysis}

The selection of homologous points in turbomachinery allows the identification of an equivalent set of design points. The idea behind this approach is to obtain equivalent working conditions (rotational speed, pressure ratio and mass flow) of a micro scale centrifugal compressor when using different working fluids. If the fluid flowing through a given turbomachine is replaced with a different fluid of different density, $\rho$ or viscosity, $\mu$, the performance of the machine will be affected; the per formance changes may be easily predicted by using dimensional ana lysis. Furthermore, the use of dimensional analysis applied to turbo machines working with different fluids has the advantage of making straightforward the determination of the operating points where the efficiency is expected to be the same. Because of dynamical similarity, efficiency changes will depend only on Reynolds number. Hence, the calculation of these points provides the new working conditions to characterize the efficiency increases or decreases on account solely of fluid properties.

The set of homologous points is obtained by means of dimensional 
analysis. The following analysis is particularized to radial compressors but could be easily extended to any generic turbomachine. For a better understanding, expressions below assume that fluids behavior are per fect gases, which seems reasonable due to the range of operation se lected. Nevertheless, the calculations were carried out using real gas properties taken from REFPROP database [26]. Fig. 1 shows an en thalpy entropy diagram representing the polytropic process through a single stage adiabatic centrifugal compressor. Stations 1 and 2 represent fluid conditions at the impeller inlet and outlet respectively, while station 3 is taken at the diffuser exit.

Eq. (1) holds the isentropic efficiency of the compression line de picted in Fig. 1, where $\Delta h_{0 \mathrm{~s}}$ is the ideal specific work and $\Delta h_{0}$ the actual specific work.

$\eta=\frac{h_{03 s}-h_{01}}{h_{03}-h_{01}}=\frac{\Delta h_{0 s}}{\Delta h_{0}}$

The parameters $\Delta h_{0 s}$ and $\eta$ can be expressed in terms of control vari ables (mass flow rate $\dot{m}$ and rotational speed $N$ ), geometric variables (diameter $D$ ), fluid properties (specific heat ratio $\gamma$, dynamic viscosity $\mu$ and gas constant $R$ ) and stagnation fluid pressure and temperature entry conditions $\left(p_{01}, T_{01}\right)$.

Dimensional analysis is the formal procedure whereby the group of variables representing some physical situation is reduced to a smaller number of dimensionless groups. Eq. (2) expresses two separate func tional relationships, each of which consist of 9 independence variables. By means of dimensional analysis Eq. (2) may be reduced to a function of 5 dimensionless groups (Eq. (3), where $a_{1}$ is the speed of sound at inlet conditions.

$\frac{\Delta h_{0 s}}{a_{1}^{2}}, \eta=f\left(\dot{m}, N, D, \gamma, \mu, R, T_{01}, p_{01}\right)$

$\frac{\Delta h_{0 s}}{a_{1}^{2}}, \eta=f\left(\frac{N D}{a_{1}}, \frac{\dot{m}}{\rho_{01} a_{1} D^{2}}, \frac{\rho_{01} N D^{2}}{\mu}, \gamma\right)$

Dynamical similarity implies that, if the dimensionless groups inside the parenthesis of Eq. (3) remain constant, the head coefficient $\Delta h_{0 s} / a_{1}^{2}$ and the efficiency $\eta$ are also constant. The first term inside the par enthesis is usually called the blade Mach number because $N D$ is pro portional to blade speed; the second one corresponds to the flow Mach number; the third is the generalized expression of Reynolds number and the last one is the ratio of specific heat capacities of the fluid.

The efficiency of a given turbomachine, a micro scale centrifugal compressor in this case, working with two different fluids will remain constant provided that all the dynamical similarity conditions are ful filled. The same could be said about the efficiency of two similar tur bomachines of different size. Whenever there is a change in one of the dimensionless groups of Eq. (3) the efficiency will change accordingly. More specifically, assuming that the influence of $\gamma$ is small and that the two Mach numbers remain constant, the efficiency will vary according to Reynolds number changes. This variation is studied further in Sec tion 2.2. As it has been mentioned, the present section deals with the calculation of the rotational speeds, compression ratios and mass flows that fulfill the similarity of the head coefficient and of the two Mach numbers specified in Eq. (3).

Starting from the definition of inlet total enthalpy (Eq. (4), the total static temperature ratio is formulated in Eq. (6) where $M_{c_{1}}$ is the Mach number of the inlet absolute velocity $c_{1}$.

$h_{01}=h_{1}+\frac{c_{1}^{2}}{2}$

$\frac{T_{01}}{T_{1}}=1+\frac{c_{1}^{2}}{2 c_{p} T_{1}}$

$\frac{T_{01}}{T_{1}}=1+\frac{\gamma-1}{2} M_{c_{1}}^{2}$
Referring to the isentropic line 0103 s represented in Fig. 1, the isen tropic stagnation enthalpy rise can be written as:

$\Delta h_{0 s}=c_{p} T_{01}\left[\left(\frac{p_{03}}{p_{01}}\right)^{\frac{\gamma-1}{\gamma}}-1\right]$

The head coefficient stands:

$\frac{\Delta h_{0 s}}{a_{1}^{2}}=\frac{\gamma R}{\gamma-1} \frac{T_{01}}{\gamma R T_{1}}\left[\left(\frac{p_{03}}{p_{01}}\right)^{\frac{\gamma-1}{\gamma}}-1\right]$

Replacing Eq. (6) in Eq. (8):

$\frac{\Delta h_{0 s}}{a_{1}^{2}}=\frac{1+\frac{\gamma-1}{2} M_{c_{1}}^{2}}{\gamma-1}\left[\left(\frac{p_{03}}{p_{01}}\right)^{\frac{\gamma-1}{\gamma}}-1\right]$

If the head coefficient $\Delta h_{0 s} / a_{1}^{2}$ is kept constant for two different fluids, then:

$\frac{1+\frac{\gamma-1}{2} M_{c_{1}}^{2}}{\gamma-1}\left[\left(\frac{p_{03}}{p_{01}}\right)^{\frac{\gamma-1}{\gamma}}-1\right]=\frac{1+\frac{\gamma^{\prime}-1}{2} M_{c_{1}^{\prime}}^{2}}{\gamma^{\prime}-1}\left[\left(\frac{p_{03}^{\prime}}{p_{01}^{\prime}}\right)^{\frac{\gamma^{\prime}-1}{\gamma^{\prime}}}-1\right]$

where variables denoted with a prime represent the new conditions to calculate the homologous point for a different fluid.

The new compression ratio $\left(p_{03}^{\prime} / p_{01}^{\prime}\right)$ of any compressor fulfilling dynamic similarity conditions after changing the working fluid is set in Eq. (11). It is worth noting that $M_{c_{1}}=M_{c_{1}^{\prime}}$ because of the dynamic si milarity.

$\frac{p_{03}^{\prime}}{p_{01}^{\prime}}=\left\{1+\frac{\gamma^{\prime}-1}{\gamma-1} \frac{1+\frac{\gamma-1}{2} M_{c_{1}}^{2}}{1+\frac{\gamma^{\prime}-1}{2} M_{c^{\prime}}^{2}}\left[\left(\frac{p_{03}}{p_{01}}\right)^{\frac{\gamma-1}{\gamma}}-1\right]\right\}^{\frac{\gamma^{\prime}-1}{\gamma^{\prime}}}$

Additionally, Eq. (12) for choked inlet flows $\left(M_{c_{1}}=M_{c_{1}^{\prime}}=1\right)$ is the same expression that was obtained by Heaton et al. in a paper where a choked turbine was studied [27].

$\frac{p_{03}^{\prime}}{p_{01}^{\prime}}=\left\{1+\frac{\gamma^{\prime}-1}{\gamma-1} \frac{\gamma+1}{\gamma^{\prime}+1}\left[\left(\frac{p_{03}}{p_{01}}\right)^{\frac{\gamma-1}{\gamma}}-1\right]\right\}^{\frac{\gamma^{\prime}-1}{\gamma^{\prime}}}$

The analysis must be completed by calculating in the same way the new rotational speed $N^{\prime}$ and the new mass flow $\dot{m}^{\prime}$. Coming back to the dynamical similarity Eq. (3) and stating that the blade Mach number is

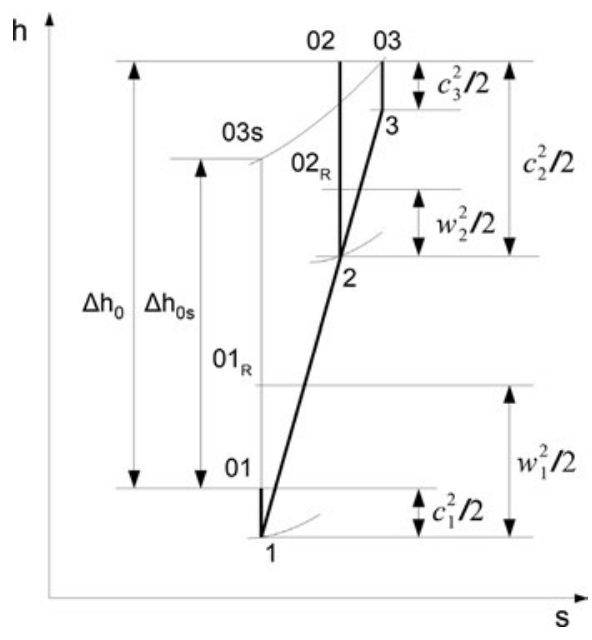

Fig. 1. Polytropic process in a centrifugal compressor. 
constant, Eq. (13) holds:

$\frac{N D}{a_{1}}=\frac{N^{\prime} D^{\prime}}{a_{1}^{\prime}}$

If the compressor size remains constant $\left(D=D^{\prime}\right)$, the new rotational speed is obtained in Eq. (14).

$N^{\prime}=N \frac{a_{1}^{\prime}}{a_{1}}=N \frac{\sqrt{\gamma^{\prime} R^{\prime} T_{1}^{\prime}}}{\sqrt{\gamma R T_{1}}}$

Finally, the flow Mach number holds:

$\frac{\dot{m}}{\rho_{01} a_{1} D^{2}}=\frac{\dot{m}}{\frac{p_{01}}{R T_{01}} \sqrt{\gamma R T_{1}} D^{2}}$

Replacing Eq. (6) in Eq. (15) and considering that the flow Mach number is kept constant for two different fluids, the following equality can be stated:

$\dot{m} \frac{\sqrt{R T_{1} / \gamma}}{p_{01} D^{2}}\left(1+\frac{\gamma-1}{2} M_{c_{1}}^{2}\right)=\dot{m}^{\prime} \frac{\sqrt{R^{\prime} T_{1}^{\prime} / \gamma^{\prime}}}{p_{01}^{\prime} D^{\prime 2}}\left(1+\frac{\gamma^{\prime}-1}{2} M_{c_{1}}^{\prime 2}\right)$

Considering again that the compressor size is constant, the new mass flow for the homologous point is obtained in Eq. (17).

$\dot{m}^{\prime}=\dot{m} \frac{p_{01}^{\prime}}{p_{01}} \sqrt{\frac{R T_{1} / \gamma}{R^{\prime} T_{1}^{\prime} / \gamma^{\prime}}} \frac{\left(1+\frac{\gamma-1}{2} M_{c_{1}}^{2}\right)}{\left(1+\frac{\gamma^{\prime}-1}{2} M_{c_{1}^{\prime}}^{2}\right)}$

Eqs. (11), (14) and (17) are used to find the compression ratio, rota tional speed and mass flow of the new equivalent points obtained after changing the working fluid. The two homologous points will have the same isentropic efficiency provided that their Reynolds numbers are also the same. Whenever there is a change in Reynolds number (be cause of kinematic viscosity variations) the efficiency will vary ac cordingly, as discussed in the next subsection.

\subsection{Reynolds number dependent efficiency correction methods}

Once the methodology to characterize new homologous points when using a different working fluid has been established, it is essential to determine the efficiency variations of the micro scale centrifugal compressors. Thanks to this methodology, efficiency variations only depend on Reynolds number changes. Hence, it should be ensured both an appropriate choice of $R e$ and a correlation to predict the real per formance of the compressor. There are quite a few methods to obtain the above mentioned influence on turbomachinery efficiency. Four of them have been reviewed in this paper for comparative purposes.

The choice of the appropriate $R e$ for radial machines requires an idea of where the highest viscous losses take place. As stated by Cumpsty [28], the most serious viscous loss source is likely to be at or near the impeller outlet, largely because the passage is narrowest there. A proper $R e$ could be therefore expressed as shown in Eq. (18), where $u_{2}$ is the impeller tip speed, $b_{2}$ is the impeller tip width at the impeller exit and $v$ is the kinematic viscosity.

$R e_{b 2}=\frac{u_{2} b_{2}}{v}$

The first efficiency correction method considered is the one proposed by Dunham and Came [29]. This empirical formula provides an straight forward estimation of the efficiency change by means of $R e_{b 2}$. Eq. (19) shows this expression, where $\eta_{0}$ is the efficiency when $R e=R e_{b 2,0}$.

$\Delta \eta=\left(1-\eta_{0}\right)+\left[1-\left(\frac{R e_{b 2,0}}{R e_{b 2}}\right)^{1 / 5}\right]$

Strub et al. [30] have devised correlation procedures for assessing Reynolds number effects in radial compressors. Near the best efficiency point the portion of the Reynolds independent losses can be represented by a constant fraction of 0.3 . The dependence of loss on Reynolds number is assumed to follow the same form as the flow in roughened pipes through a representative value of the friction coefficient $\lambda$ ac cording to the Moody diagram. The proposed formula for efficiency correction is given in Eq. (20), where $\lambda_{\text {crit }}$ is the value of the pipe flow friction factor attained as $R e_{b 2}$ tends to infinity, not to be confused with the critical value at the transition from laminar to turbulent flow.

$\Delta \eta=-\left(1-\eta_{0}\right)+\left[1-\frac{0.3-0.7 \frac{\lambda}{\lambda_{\text {crit }}}}{0.3-0.7 \frac{\lambda_{0}}{\lambda_{\text {crit }}}}\right]$

Nevertheless, most recent literature promote the use of a chord based Reynolds number. This is the case of Casey and Robinson [31], who claim that this approach is appropriate because of the boundary layers on the blade surface are thin in internal flows and do not become fully developed as in a pipe flow. Eq. (21) expresses this Reynolds number based on the inlet relative velocity at mid span $w_{1}$ and the chord length $c$ (length of the flow path).

$R e_{c}=\frac{w_{1} c}{v}$

Following this approach, Dietmann and Casey [32] obtained a gen eralized efficiency correlation based on an empirical coefficient $B_{\text {ref }}$ which differs for different machine types (Eq. (22)). The adjustment of this coefficient notably agrees with the flow coefficient of both original test data and analytic calculations. Another difference found in this efficiency characterization method is the friction coefficient $\left(c_{f}\right)$ ana logy: flow along a flat plate instead of pipe flow. Two friction coeffi cients (laminar and turbulent) are calculated using $R e_{c}$ definition and combined by means of a blending function. This improvement refines the transition zone between laminar and smoothly turbulent region, which is the region of special interest in this work. One may note that this procedure corrects polytropic efficiency instead of isentropic effi ciency.

$\Delta \eta_{p}=-B_{r e f}(\Phi) \frac{\Delta c_{f}}{c_{f, 0}}$

Finally, Pelz and Stonjek have developed a new scaling method for the compressors efficiency based on a physical formulation with only minor assumptions [33,34]. According to their calculations, the rotor/im peller and the volute/stator have to be considered separately in a physically based method. That is why an $i$ th Reynolds number needs to be defined in order to quantify independently both friction phenomena. Eq. (23) expresses this $R e_{i}$ as a function of the characteristic relative velocity $w_{\text {char }, i}$ and characteristic length $L_{i}$.

$R e_{i}=\frac{w_{\text {char }, i} L_{i}}{\nu}$

The efficiency correction procedure according to Pelz and Stonjek is presented in Eq. (24). The same analogy of flat plate is used to de termine the friction coefficient in both impeller and diffuser. Then, a geometry dependent function weights both coefficients in order to ob tain the overall $c_{f}$.

$\Delta \eta=-\left(1-\eta_{0}\right) \frac{\Delta c_{f}}{c_{f, 0}}$

\subsection{Numerical model}

The numerical model presented below has been developed using the commercial software ANSYS CFX 19. Computational fluid dynamics (CFD) analysis is the method proposed in this work to validate the homologous points choice for the selected fluids and the application of the Reynolds number dependent efficiency correction methods. The use of CFD in small scale turbomachinery have been validated based on the 
good agreement between numerical and experimental results predicting the main flow features within the compressor $[35,36]$.

The geometry used in this calculation corresponds to a left handed micro scale centrifugal compressor which will be soon available for future experimentation within an instrumented test rig. Table 1 shows its main specifications for standard conditions ISO 8778 (293.15 K and $100 \mathrm{kPa}$ ). One may note that the impeller diameter is well below $30 \mathrm{~mm}$ and the chord based $R e$ lays in the laminar turbulent transition region. The stage is formed by an impeller diffuser passage with an straight inlet duct. The volute has not been included in this study in order to reduce the computational cost and, thus, the outlet boundary is set at the end of the diffuser. Both the impeller blades and the vaneless dif fuser have been designed according to the manufacturer specifications [37] using ANSYS BladeGen together with a predesign obtained by means of ANSYS Vista CCD.

Meshing and grid processing have been carried out with ANSYS TurboGrid. Automatic Topology Meshing (ATM) is a new method in tegrated in ANSYS which results in high quality anisotropic structured hexahedral mesh. Its main advantage is to ensure mesh quality even when blade shape changes from hub to tip. Fig. 2 depicts the meshed compressor model with the shroud hidden where a change in grey shade shows the impeller and diffuser geometries. A grid independence study has been carried out to ensure that the results are not mesh de pendent. Following the procedure published by Celik et al. [38], three different meshes has been studied with 1.2, 2.8 and 6.6 millions cells respectively. Grid convergence index (GCI) provides a consistent parameter to determine the error band on the grid convergence solu tion. GCI from fine to intermediate $\left(\mathrm{GCI}_{12}\right)$ and from intermediate to coarse grid $\left(\mathrm{GCI}_{23}\right)$ have been maintained below $0.5 \%$ for pressure ratio and isentropic efficiency key functions in design point conditions. These values are well below the acceptable convergence threshold (2 3\%). Besides, monotonic convergence is ensured $(\mathrm{R}=0.37)$ and the ex pression $G C I_{23} \simeq r^{p} G C I_{12}$ is fulfilled with an uncertainty of $0.14 \%$, where $r$ is the grid refinement factor and $p$ the observed rate of con vergence. All this guarantees the achievement of the asymptotic range and, therefore, grid insensitive results.

Three different computational domains are built: inlet duct, im peller and vaneless diffuser. The inlet duct and the vaneless diffuser are set as stationary domains, while the impeller is a rotating domain with a counter rotating shroud. Frozen rotor approach is employed in inter faces between stationary and rotating domains. Solid to fluid interfaces are set as no slip walls with smooth roughness. Total inlet pressure, temperature and outlet mass flow are set as initial boundary conditions. Redlich Kwong equations of state characterize the working fluid used (air) by means of REFPROP database [26], included in ANSYS software. The turbulence model selected is the $\mathrm{k}-\omega$ Shear Stress Transport (SST) [39], which combines the advantages of $k-\epsilon$ and $k-\omega$ models, with an initial moderate turbulence intensity of $5 \%$ at the inlet boundary. This model is particularly well suited to this application due to its high ac curacy and robustness simulating laminar turbulent flow transition in boundary layers [40]. Non dimensional distance $\left(\mathrm{y}^{+}\right)$of the first node after the node of the wall is kept below or equal to the unity in almost all the surfaces as recommended in CFX user's manual. Hence, a reliable boundary layer is obtained ensuring an appropriate near wall treat ment.

\section{Results and discussion}

The present section intends to present a reliable characterization of the reference micro scale centrifugal compressor following the pro posed methodology. First, a validation study is carried out to verify the Reynolds number dependent efficiency correlations approach for homologous points. Then, the reference MSCC performance is analyzed using different non conventional working fluids with good potential for B HRB and ORC small scale power cycles. The results obtained show interesting performance predictions for this type of turbomachines working in conditions far from those of the design point.

\subsection{Micro scale air centrifugal compressor efficiency characterization}

First, a Reynolds number dependent efficiency characterization is carried out for the reference MSCC described in Section 2.3 with air as working fluid. Instead of varying explicitly the Reynolds number, total inlet pressure is selected as the control variable in this study. If the inlet temperature is maintained constant the increase in pressure leads to a linear decrease in kinematic viscosity and, thus, Reynolds number is consequently increased.

Following the methodology proposed in Section 2, a set of homo logous points has been identified to conduct this analysis. For each total inlet pressure, the homologous mass flow and rotational speed are calculated to define new design working conditions. These points en sure that Reynolds number is the parameter which has the highest impact on compressor efficiency variation. This efficiency variation is estimated by means of the four efficiency correction methods pre viously described, together with the numerical simulation. One may note the beneficial use of total inlet pressure as control variable due to the different definition of Reynolds number employed in each method.

According to the manufacturer specifications, the reference turbo compressor at its nominal design point performs a compression ratio of 1.4 and $7 \mathrm{~g} / \mathrm{s}$ air mass flow at 225,000 $\mathrm{min}^{-1}$ with an overall efficiency of $63 \%$ [37]. Motor and converter efficiencies represent a $92 \%$ and $90.6 \%$ [41], respectively. As the isentropic efficiency is the output discussed in this study, the nominal isentropic efficiency is $0.63 /(0.92 \cdot 0.906)=75.6 \%$.

Fig. 3 shows the isentropic efficiency change vs. the total inlet pressure taking air as working fluid of the reference MSCC at $293.15 \mathrm{~K}$. Inlet vacuum conditions are taken into account because they are esaily achievable with air in experimentation. Pelz and Stonjek and Dunham and Came seem to be the less accurate methods if the CFD simulation is taken as a reference. On the other hand, Strub et al. and Dietmann and Casey methods achieve good results, being the latter the most accurate throughout the studied range of pressures. One may note that this method estimate polytropic efficiency and, therefore, needs to be transformed in isentropic efficiency. For micro scale approach, it can be stated that the use of chord based Reynolds number and a blending function to obtain a Reynolds number laminar turbulent ensures that efficiency correction is in accordance with numerical simulations.

As explained in Section 2.2, each efficiency correction method has its own Reynolds number approach. Table 2 gathers these different definitions of Reynolds number at vacuum conditions, atmospheric pressure and overpressure. It should be noted that these differences are a consequence of their characteristic dimension and velocity.

\subsection{Performance prediction for non conventional fluids}

Once the proposed methodology has been validated with the re ference micro scale centrifugal compressor using air as working fluid, this subsection deals with the performance prediction for non conven tional fluids. From now on the method employed to calculate efficiency

Table 1

Reference micro-scale centrifugal compressor data for standard conditions ISO 8778.

$\begin{array}{lc}\text { No. main blades (\#) } & 7 \\ \text { No. splitted blades (\#) } & 7 \\ \text { Diffuser type } & \text { Vaneless } \\ \text { Impeller diameter (mm) } & 21 \\ \text { Flow coefficient (-) } & 0.068 \\ \text { Head coefficient (-) } & 0.486 \\ \text { Tip Mach number (-) } & 0.721 \\ \text { Specific speed (-) } & 0.794 \\ \text { Chord Reynolds-number (-) } & 7.72 \times 10^{4}\end{array}$




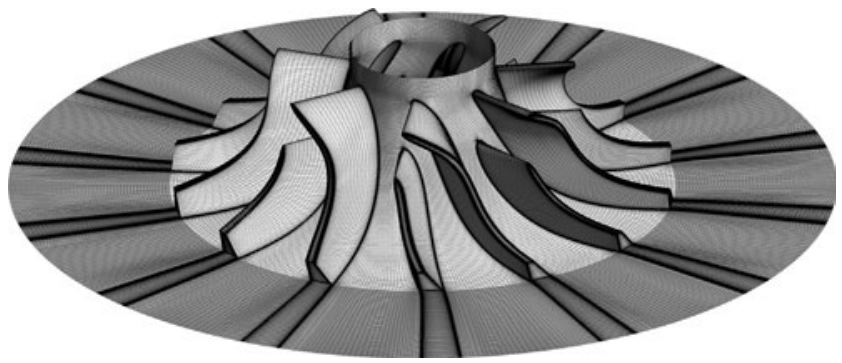

Fig. 2. Meshed impeller and vaneless diffuser geometries.

variations is the Dietmann and Casey one, according to the conclusions drawn in the precious section. This work seeks the estimation of the performance increase (or decrease) when using alternative fluids in a MSCC for innovative power cycles. As briefly explained in the in troduction of this paper, the selected fluids for this study are those which fit well with novel thermodynamic cycles optimized for low to moderate temperatures sources $[7,24,25]$. These potential working fluids are carbon dioxide $\left(\mathrm{CO}_{2}\right)$, isobutane (R600a), propane (R290a), pentafluoroethane (R125) and sulfur hexafluoride $\left(\mathrm{SF}_{6}\right)$. Air perfor mance will be used as the reference to quantify relative variations.

Table 3 shows the main characteristics of the selected fluids. All of them except air have critical temperature above ambient temperature, which means that inside a recipient maintained at ambient temperature there is a two phase equilibrium condition at the saturation pressure. The pressure can be reduced from the saturation value to any given inlet value by means of a lamination valve located between the re cipient and the compressor entry. For the air case, the pressure con sidered in Table 3 is atmospheric pressure. For the other fluids, the properties are taken at the saturation pressure at $293.15 \mathrm{~K}$.

The isentropic efficiency prediction of the reference MSCC using the selected working fluids is again employed in Fig. 4 as a function of total inlet pressure. The same Reynolds number variation approach of Sec tion 3.1 is deployed in this study. It can be noted that all non con ventional fluids achieve higher efficiency than air. Substantial differ ences are found in pressures near atmospheric conditions because the $R e$ values belong to the laminar turbulent transition region. The use of isobutane instead of air as working fluid may increase isentropic effi ciency in 3 percentage points. This increase is basically due to its par ticular thermophysical properties when the MSCC is operating in the new calculated set of working points. It should be also observed that isobutane inlet pressure cannot be higher than its saturation pressure, so its curve ends before reaching 3 bar. Propane, $\mathrm{SF}_{6}$ and R125 lead to notably efficiency variations throughout the whole range studied. Their
Table 2

Different Reynolds-number definitions for different air inlet pressures at $293.15 \mathrm{~K}$.

\begin{tabular}{lcccc}
\hline Inlet pressure & $R e_{b 2}$ & $R e_{c}$ & $R e_{i}^{i m p}$ & $R e_{i}^{\text {dif }}$ \\
\hline 0.5 bar & $8.38 \times 10^{3}$ & $3.96 \times 10^{4}$ & $2.29 \times 10^{3}$ & $1.79 \times 10^{4}$ \\
1.013 bar & $1.68 \times 10^{4}$ & $7.95 \times 10^{4}$ & $4.59 \times 10^{3}$ & $3.59 \times 10^{4}$ \\
2.5 bar & $4.41 \times 10^{4}$ & $1.96 \times 10^{5}$ & $1.13 \times 10^{4}$ & $8.85 \times 10^{4}$ \\
4 bar & $6.63 \times 10^{4}$ & $3.14 \times 10^{5}$ & $1.80 \times 10^{4}$ & $1.42 \times 10^{5}$ \\
\hline
\end{tabular}

Table 3

Main physical properties of the potential working fluids at $293.15 \mathrm{~K}$.

\begin{tabular}{lccccc}
\hline Fluid & $p_{\text {sat }}$ (bar) & $\rho\left(\mathrm{kg} / \mathrm{m}^{3}\right)$ & $a_{1}(\mathrm{~m} / \mathrm{s})$ & $T_{\text {crit }}(\mathrm{K})$ & $p_{\text {crit }}$ (bar) \\
\hline Air $(p=1.013$ bar $)$ & - & 1.2 & 334.3 & 132.5 & 37.9 \\
$\mathrm{CO}_{2}$ & 57.3 & 194.2 & 196.1 & 304.1 & 73.8 \\
Isobutane & 3.0 & 7.9 & 198.2 & 407.8 & 36.4 \\
Propane & 8.3 & 18.0 & 217.2 & 369.6 & 42.5 \\
R125 & 12.1 & 78.0 & 119.6 & 339.2 & 36.3 \\
$\mathrm{SF}_{6}$ & 21.0 & 191.5 & 95.0 & 318.7 & 37.6 \\
\hline
\end{tabular}

asymptotic value ends in an efficiency increase of 4 percentage points than using atmospheric air. Efficiency variation using carbon dioxide is similar to the one using air. As a general comment, the effect of Rey nolds number on the reference centrifugal compressor efficiency is particularly interesting in pressure ranges near to atmospheric condi tions, whereas the differences are lower at high inlet pressures.

In addition to Reynolds number dependent efficiency changes, it is interesting to obtain the rest of the compressor working characteristics: load power, mass flow, rotational speed and pressure ratio. Fig. 5 shows the compressor power requirements (including mechanical losses) vs. the total inlet pressure. The maximum allowed power according to the compressor manufacturer is $1 \mathrm{~kW}$ [37]. One may appreciate that all the potential working fluids selected fit well with the compressor properties in the pressure range specified, as the power requirements are below $1 \mathrm{~kW}$. The only fluid calculated for vacuum conditions is air, explained in the previous subsection. Isobutane, $\mathrm{SF}_{6}$ and $\mathrm{R} 125$ are those with the minimum power requirements. These low values mean that larger compressors with higher power consumption should be required for high powered thermodynamic cycles in comparison to air. Never theless, the power requirements obtained for the low powered cycles addressed in this paper help to the minimization of the compression power consumption.

When the power load for a given fluid is increased, the mass flow varies accordingly as represented in Fig. 6. The two fluids with the

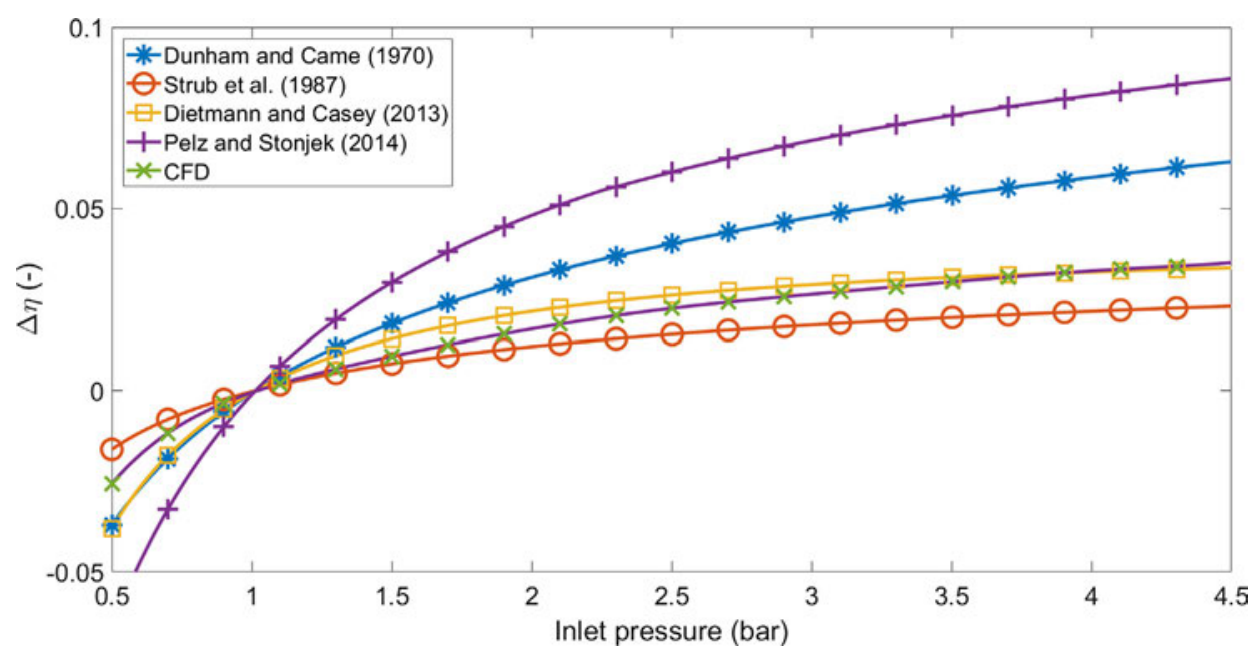

Fig. 3. Comparison of efficiency variation methods for the reference MSCC at different air pressures and $293.15 \mathrm{~K}$. 




Fig. 4. Comparison of efficiency variation for the reference MSCC using the selected fluids at different inlet pressures and $293.15 \mathrm{~K}$.

lower load power requirements $\left(\mathrm{SF}_{6}\right.$ and $\left.\mathrm{R} 125\right)$ present a significant increase in mass flow. The rest of fluids show similar patterns than air. One may note that isobutane requires low mass flow rate (similar to air) although a substantial reduction in power requirements is achieved. This, together with the isentropic efficiency increase and the inlet pressure range makes this working fluid very interesting for the purpose of this paper.

Finally, rotational speeds and pressure ratio values of the homo logous points calculated for the selected fluids are reflected in Table 4. Huge variations of rotational speeds among the different fluids can be appreciated but with narrow ranges for the pressure values chosen. The effects of speed of sound variations with inlet pressure on compressor performance can be appreciated in these parameters. Fortunately, these values of rotational speeds are below the $225,000 \mathrm{~min}^{-1}$, and, thus, under the reference MSCC allowed limit. Besides, minor differences are found in pressure ratio which ensures a maintained performance in dependently of the fluid selected.

\section{Summary and conclusions}

The present paper has studied the effect of Reynolds number on micro scale centrifugal compressors performance with working fluids suited to novel high efficiency power cycles. The proposed metho dology begins with the calculation of a set of homologous points by means of dimensional analysis where efficiency changes only depend on
Reynolds number. Then, various Reynolds number dependent effi ciency correction methods were applied in the calculated set of new working points and validated by means of numerical simulation.

In the light of the results of this research, the following conclusions are drawn.

- The efficiency correction procedure developed by Dietmann and Casey [32] and their proposal of using a chord based Reynolds number has been shown to be consistent with numerical simulation when an air micro scale centrifugal compressor operates at the working points calculated.

- Beneficial effects on the isentropic efficiency of the reference micro scale centrifugal compressor have been identified when using the selected non conventional fluids. In particular, isobutane provides the higher increase in efficiency ( 34 percentage points) followed by a similar tendency with propane, R125 and $\mathrm{SF}_{6}$.

- Increases in Reynolds number, i.e. total inlet pressure, could also be put to profit to design turbomachinery even smaller than that with air as working fluid.

- Using the selected non conventional working fluids instead of air, the compressor power requirements decrease. This allows a future experimentation stage with the reference compressor working with all the fluids selected.

- Rotational speed ranges vary greatly from one fluid to another, re maining below the maximum rotational speed of the reference

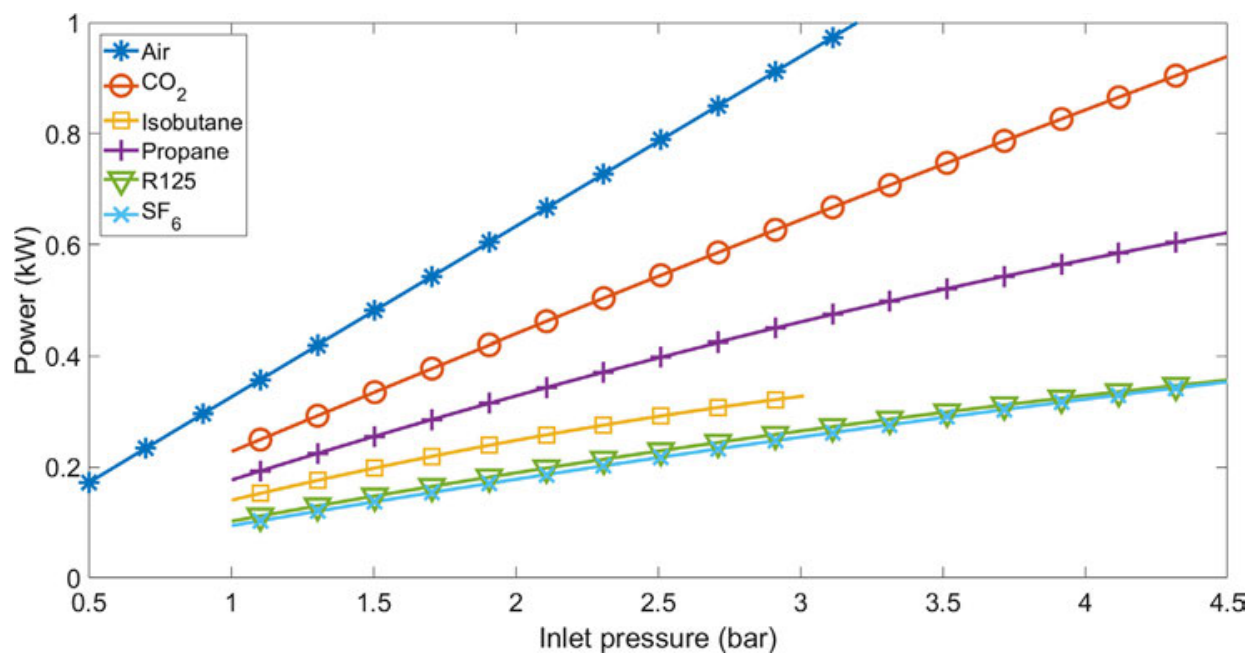

Fig. 5. Comparison of compressor load power for the reference MSCC using the selected fluids at different inlet pressures and $293.15 \mathrm{~K}$. 


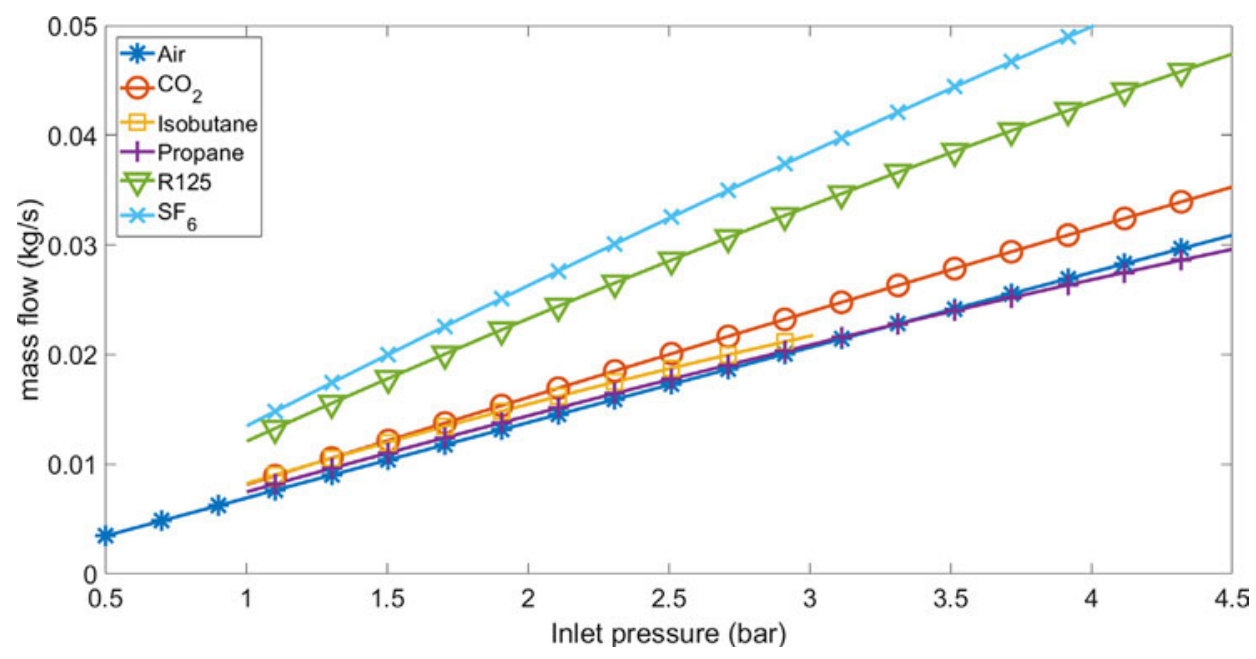

Fig. 6. Comparison of mass flow for the reference MSCC using the selected fluids at different inlet pressures and $293.15 \mathrm{~K}$.

Table 4

Rotational speed ranges and pressure ratio for the working point calculated for the reference MSCC using the non-conventional fluids selected.

\begin{tabular}{lccc}
\hline Fluid & $p$ range (bar) & $N^{\prime}\left(10^{3} \min ^{1}\right)$ & $p_{03}^{\prime} / p_{01}^{\prime}(-)$ \\
\hline Air & $0.5-4.5$ & 225 & 1.40 \\
$\mathrm{CO}_{2}$ & $1.0-4.5$ & $173-175$ & $1.37-1.38$ \\
Isobutane & $1.0-3.0$ & $131-139$ & $1.32-1.33$ \\
Propane & $1.0-4.5$ & $154-163$ & $1.33-1.34$ \\
R125 & $1.0-4.5$ & $92-98$ & $1.32-1.33$ \\
$\mathrm{SF}_{6}$ & $1.0-4.5$ & $85-88$ & $1.31-1.32$ \\
\hline
\end{tabular}

micro scale centrifugal compressor working with air. Besides, small variations in pressure ratio ensure a consistent behavior when changing the working fluid.

\section{Acknowledgments}

This research has been supported by the Spanish Ministerio de Economía y Competividad under the project of reference number ENE2015 70515 C2 2 R.

Special thanks to Fundación IBERDROLA, that has helped to this research through its Research Grants for Energy and Environment 20182019.

Authors also acknowledge Universidad Politécnica de Madrid for the support given to this research by means of Programa Propio $I+D+i$.

\section{References}

[1] Martinez S, Michaux G, Salagnac P, Bouvier J-L. Micro-combined heat and power systems (micro-chp) based on renewable energy sources. Energy Convers Manage 2017; 154:262-85.

[2] Aichmayer L, Garrido J, Laumert B. Scaling effects of a novel solar receiver for a micro gas-turbine based solar dish system. Sol Energy 2018;162:248-64.

[3] Ruzzenenti F, Bravi M, Tempesti D, Salvatici E, Manfrida G, Basosi R. Evaluation of the environmental sustainability of a micro CHP system fueled by low-temperature geothermal and solar energy. Energy Convers Manage 2014;78:611-6.

[4] Guo F, Dong Y, Lv Z, Fan P, Yang S, Dong L. Kinetic behavior of biomass under oxidative atmosphere using a micro-fluidized bed reactor. Energy Convers Manage 2016;108:210-8

[5] Badami M, Chicco G, Portoraro A, Romaniello M. Micro-multigeneration prospects for residential applications in italy. Energy Convers Manage 2018;166:23-36.

[6] Selinger-Lutz O, Pratidino G, Hollinger R, Fischer D, Koch B, Wittwer C. Flexibility assessment of a pool of residential micro combined heat and power systems. Energy Convers Manage 2018;172:228-36.

[7] Rovira A, Rubbia C, Valdés M, Martínez-Val JM. Thermodynamic cycles optimised for medium enthalpy units of concentrating solar power. Energy 2014;67:176-85.

[8] Valdés M, Abbas R, Rovira A, Martín-Aragón J. Thermal efficiency of direct, inverse and $\mathrm{sCO} 2$ gas turbine cycles intended for small power plants. Energy 2016;100:66-72.
[9] Reyes-Belmonte MA, Sebastián A, González-Aguilar J, Romero M. Performance comparison of different thermodynamic cycles for an innovative central receiver solar power plant. AIP Conference Proceedings 2017;1850(1):160024.

[10] Reyes-Belmonte M, Sebastián A, Spelling J, Romero M, González-Aguilar J. Annual performance of subcritical rankine cycle coupled to an innovative particle receiver solar power plant. Renew Energy 2019;130:786-95.

[11] Casey M, Krähenbuhl D, Zwyssig C. The design of ultra-high-speed miniature centrifugal compressors. In: Proceedings of the 10th European Conference on Turbomachinery, Fluid Dynamics and Thermodynamics; 2013.

[12] Dixon S, Hall C. Chapter 2 - dimensional analysis: similitude. In: Dixon S, Hall C, editors. Fluid mechanics and thermodynamics of turbomachinery. 7th ed.Boston: Butterworth-Heinemann; 2014. p. 39-67.

[13] Tiainen J, Jaatinen-Värri A, Grönman A, Fischer T, Backman J. Loss development analysis of a micro-scale centrifugal compressor. Energy Convers Manage 2018; 166:297-307.

[14] Jubori AA, Daabo A, Al-Dadah RK, Mahmoud S, Ennil AB. Development of microscale axial and radial turbines for low-temperature heat source driven organic rankine cycle. Energy Convers Manage 2016;130:141-55.

[15] Jubori AMA, Al-Dadah R, Mahmoud S. An innovative small-scale two-stage axial turbine for low-temperature organic rankine cycle. Energy Convers Manage 2017; $144: 18-33$.

[16] Daabo AM, Jubori AA, Mahmoud S, Al-Dadah RK. Parametric study of efficient small-scale axial and radial turbines for solar powered Brayton cycle application. Energy Convers Manage 2016;128:343-60.

[17] Muñoz-Antón J, Rubbia C, Rovira A, Martínez-Val JM. Performance study of solar power plants with $\mathrm{CO} 2$ as working fluid. a promising design window. Energy Convers Manage 2015;92:36-46.

[18] Reyes-Belmonte MA, Sebastián A, Romero M, González-Aguilar J. Optimization of a recompression supercritical carbon dioxide cycle for an innovative central receiver solar power plant. Energy 2016;112:17-27.

[19] Manjunath K, Sharma O, Tyagi S, Kaushik S. Thermodynamic analysis of a supercritical/transcritical CO2 based waste heat recovery cycle for shipboard power and cooling applications. Energy Convers Manage 2018;155:262-75.

[20] Yang S, Chen S, Chen X, Zhang X, Hou Y. Study on the coupling performance of a turboexpander compressor applied in cryogenic reverse Brayton air refrigerator. Energy Convers Manage 2016;122:386-99.

[21] Schiffmann J, Favrat D. Design, experimental investigation and multi-objective optimization of a small-scale radial compressor for heat pump applications. Energy 2010;35(1):436-50.

[22] Javed A, Arpagaus C, Bertsch S, Schiffmann J. Small-scale turbocompressors for wide-range operation with large tip-clearances for a two-stage heat pump concept. Int J Refrig 2016;69:285-302.

[23] Zhao D, Xu L, Huangfu Y, Dou M, Liu J. Semi-physical modeling and control of a centrifugal compressor for the air feeding of a PEM fuel cell. Energy Convers Manage 2017;154:380-6.

[24] Rovira A, Muñoz M, Sánchez C, Martínez-Val JM. Proposal and study of a balanced hybrid Rankine-Brayton cycle for low-to-moderate temperature solar power plants. Energy 2015;89:305-17.

[25] Muñoz M, Rovira A, Sánchez C, Montes MJ. Off-design analysis of a hybrid RankineBrayton cycle used as the power block of a solar thermal power plant. Energy 2017;134:369-81.

[26] Lemmon E, Huber M, McLinden M. Nist standard reference database 23: Reference fluid thermodynamic and transport properties-refprop, version 9.1, national institute of standards and technology, standard reference data program, gaithersburg, See http://www.nist.gov/srd/nist23.cfm.

[27] Heaton TR, Slivka WR, Westra LF. Cold-air investigation of a turbine with non twisted rotor blades suitable for air cooling. Tech. Rep. NACA RM E52A25; 1952.

[28] Cumpsty N. Compressor aerodynamics. v. 10. Krieger Pub.; 2004. Ch. 1.

[29] Dunham J, Came PM. Improvements to the Ainley-Mathieson method of turbine 
performance prediction. J Eng Power 1970;92(3):252-6.

[30] Strub RA, Bonciani L, Borer CJ, Casey MV, Cole SL, Cook BB, Kotzur J, Simon H, Strite MA, et al. Influence of the reynolds number on the performance of centrifugal compressors. J Turbomach 1987;109(4):541-4.

[31] Casey M, Robinson C. A unified correction method for reynolds number, size, and roughness effects on the performance of compressors. Proc Inst Mech Eng A: J Power Energy 2011;225(7):864-76.

[32] Dietmann F, Casey M. The effects of reynolds number and roughness on compressor performance. In: Proceedings of the 10th European conference on turbomachinery, fluid dynamics and thermodynamics; 2013.

[33] Pelz P, Stonjek S. The influence of reynolds number and roughness on the efficiency of axial and centrifugal fans - a physically based scaling method. J Eng Gas Turbines Power 2013;135(5):052601-8.

[34] Pelz P, Stonjek S. Introduction of an universal scale-up method for the efficiency of axial and centrifugal fans. In: Proceedings of ASME Turbo Expo 2014: Turbine Technical Conference and Exposition GT2014; 2014.
[35] Hazby HR, Xu L, Casey MV. Investigation of the flow in a small-scale turbocharger centrifugal compressor. Proc Inst Mech Eng A: J Power Energy 2017;231(1):3-13.

[36] Khalil KM, Mahmoud S, Al-Dadah R. Development of innovative non-repeated annular area dual stage small-scale nitrogen axial turbine for hybrid open-closed rankine cycle. Energy Convers Manage 2018;164:157-74.

[37] Celeroton, Radial turbo compressor CT-17-700 datasheet, rev06 (September 2016).

[38] Celik IB, Ghia U, Roache PJ, Freitas CJ, Coleman H, Raad PE. Procedure for estimation and reporting of uncertainty due to discretization in CFD applications. J Fluid Eng-Trans ASME 2008;130(7). 078001-078001-4.

[39] Menter FR. Two-equation eddy-viscosity turbulence models for engineering applications. AIAA J 1994;32(8):1598-605.

[40] Menter FR. Review of the shear-stress transport turbulence model experience from an industrial perspective. Int J Comput Fluid Dyn 2009;23(4):305-16.

[41] Zhao D, Daniel K, Blunier B, Zwyssig C, Dou M, Miraoui A. Design and control of an ultra high speed turbo compressor for the air management of fuel cell systems. Transportation Electrification Conference and Expo (ITEC). IEEE; 2012. p. 1-6. 